\title{
WIGNER CRYSTALLIZATION IN InGaAs/InP HETEROSTRUCTURES WITH A STRONG DISORDER ${ }^{\dagger}$
}

Gy. Kovács ${ }^{a}$, G. Remenyi ${ }^{b}$, G. Gombos ${ }^{c}$, I.G. SAvel'ev ${ }^{d}$, A.M. KReshChUK ${ }^{d}$, N. HegmaN ${ }^{e}$ AND B. PöDöR ${ }^{c}$

${ }^{a}$ Department of Low Temperature Physics, Roland Eötvös University

Puskin u. 5-7, 1088 Budapest, Hungary

${ }^{b}$ CNRS Centre de Recherches sur les très Basses Températures, 38042 Grenoble, France

${ }^{c}$ Research Institute for Technical Physics of the Hungarian Academy of Sciences

P.O. Box 76, 1325 Budapest, Hungary

${ }^{d}$ A.F. Ioffe Physico-Technical Institute of the Russian Academy of Sciences

194021 St. Petersburg, Russia

e Institute of Nuclear Research of the Hungarian Academy of Sciences

P.O.Box 51, 4001 Debrecen, Hungary

Non-linear current-voltage characteristics were observed in the range of filling factors of $0.3 \leq \nu \leq 0.4$ in a two-dimensional electron system in In GaAs/InP heterostructures with a strong disorder. The observations are explained qualitatively in terms of magnetic field induced localization and Wigner solidification.

PACS numbers: $73.20 . \mathrm{Dx}$

\section{Introduction}

The effect of the disorder on the ground state of a two-dimensional electron system (2DES) is far from being cleared. A sufficiently strong disorder can obviously destroy both the fractional quantum Hall effect (FQHE) states and the Wigner crystal (WC). Due to the disorder magnetic field induced localization takes place, thus at sufficiently low temperature the diagonal component of the resistivity $\left(Q_{x x}\right)$ diverges exponentially as the filling factor $(\nu)$ is decreased. Furthermore, $\rho_{x x}$ is thermally activated for $\nu<1$ in the strong (but not yet destructive) disorder limit. If this is the case, the strength of the disorder $\langle V\rangle$ is related to the Coulomb energy $(U)$ as $e\langle V\rangle / U \approx 1$, and the ground state of the 2DES is supposed to be a glass-like condensate (Wigner glass) [1].

thigh magnetic field experiments were done in the CNRS Laboratoire des Champs Magnétiques Intenses, Grenoble. 
In the case of a low but finite disorder, (i.e., for $e\langle V\rangle / U \ll 1$ ) activated conduction sets in a small but non-zero value of $\nu$, and the corresponding ground state of the 2DES is a WC pinned by the disorder [2-4].

We report here on the first observation of non-linear current-voltage characteristics accompanied by diverging resistivity in a 2DES in a lattice-matched InGaAs/InP heterostructure with a known and relatively high disorder. Down to $60 \mathrm{mK}$ temperature and for a filling factor of about 0.22 no signs of FQHE were seen, indicating that the disorder is rather strong. Both the $I-V$ characteristics and their temperature dependence can be qualitatively understood if we assume a disorder pinned WC.

\section{Experimental}

The lattice-matched InGaAs/InP heterostructure was grown by liquid phase epitaxy $[5,6]$. The parameters of the 2DES were $n_{\mathrm{s}}=1.2 \times 10^{11} \mathrm{~cm}^{-2}$ and $\mu=$ $3 \times 10^{4} \mathrm{~cm}^{2} \mathrm{~V}^{-1} \mathrm{~s}^{-1}$. The rather low mobility was due to the disorder, the physical origin of which was investigated in our heterostructures in detail in Refs. [7, 8]. The strength of the disorder was estimated from the Landau level broadening to be about $e\langle V\rangle=8 \div 10 \mathrm{meV}$, which is comparable in magnitude with the Coulomb interaction energy for the given 2DES concentration.

The magnetotransport experiments were done with a resistive magnet providing fields up to $23 \mathrm{~T}$. The measurements were performed from $150 \mathrm{mK}$ to $60 \mathrm{mK}$, the lowest temperature reached in a dilution refrigerator. Magnetic fields and temperatures were estimated to be correct to about $0.02 \mathrm{~T}$ and better than $10 \%$, respectively. Both dc and standard, low frequency ( $8 \mathrm{IIz}$ ) lock-in techniques were used.

\section{Results and discussion}

Both dc and ac measurements revealed that at and below $\nu=0.4$ the resistance of the sample began to increase drastically, approximately exponentially in the magnetic field, then the measurements of the longitudinal and of the Hall resistance had to be terminated because of a noisy resistance roll-off, which lead to the onset of large, out of phase components.

Figure 1 summarizes the two-terminal $I-V$ characteristics for different magnetic fields for $70 \mathrm{mK}$ temperature. In the range of filling factors $0.3 \leq \nu \leq 0.4 \mathrm{a}$ definite non-linearity occurs with a magnetic field dependent "plateau". For higher filling factors these features are absent, thus the magnetic field induced localization sets in at rather high filling, indicating again that the amplitude of the disorder potential is substantial.

Figure 2 shows the temperature dependence of the $I-V$ characteristics, revealing saturation for higher temperatures $(T \geq 90 \mathrm{mK})$ with a lower "threshold voltage". Note that this is just the opposite to saturation expected in the case of excessive electron heating. The heating of the electrons can be neglected in this regime, since the phase difference between the voltage and the current is close to $\pi / 2$, and the contribution of the current to the Joule heat almost vanishes. The 


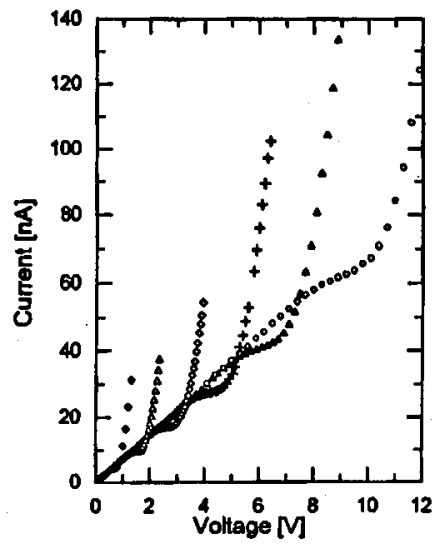

Fig. 1. $I-V$ characteristics measured by the two-point method at $T=70 \mathrm{mK}$ in magnetic fields increasing from $12 \mathrm{~T}$ to $17 \mathrm{~T}$ in steps of $1 \mathrm{~T}$ from left to right. The corresponding filling fact ${ }^{20} 9$ are $\nu^{\prime}=0.414,0.382,0.354,0.331,0.310$, and 0.292 , respectively.

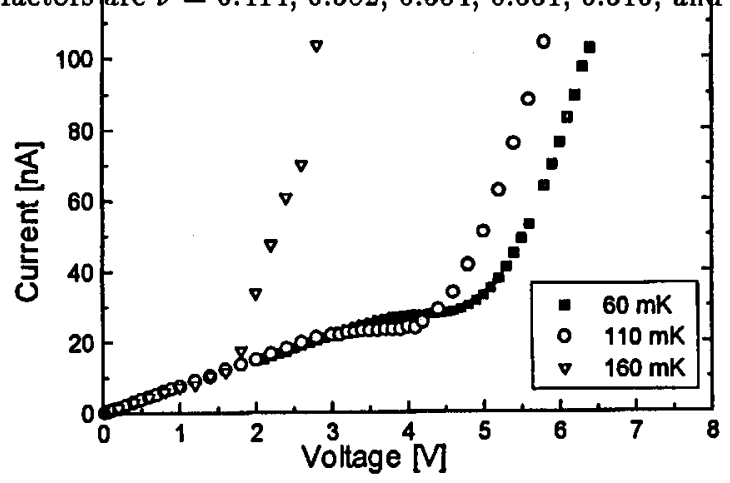

Fig. 2. $I-V$ characteristics measured at different temperatures as indicated, at the fixed value of magnetic field of $15 \mathrm{~T}$.

observed saturation behaviour is difficult to reconcile with the assumption of Anderson localization. On the other hand, it is quite analogous to the one reported in Ref. [3], where it has been considered as a signature of Wigner solidification.

The observed values of the threshold electric field, i.e., a few tens of $\mathrm{V} / \mathrm{cm}$, are larger than the value expected for an ordinary WC. A possible explanation of this difference can be based on an assumption of pinning of the crystalline electron system by the disorder. For all values of the applied temperatures, the threshold electric field is nearly proportional to the square of the magnetic field. This disorder, stronger than the usual one encountered in GaAlAs/GaAs, fully destroys the incompressible fluid state of 2DES, thus FQHE plateaux are not formed. However, the non-linear current-voltage relationship and its temperature and magnetic field dependence raises the possibility of the formation of a WC pinned by the disorder. 
In conclusion we have observed non-linear current-voltage characteristics and determined the respective threshold fields in two-terminal longitudinal resistivity of $2 \mathrm{DES}$ in InGaAs/InP heterostructures in the range of the filling factor below about 0.4 . We have suggested the possibility of the formation of a disorder pinned Wigner crystal to explain the experimental observations.

\section{Acknowledgments}

The high magnetic field experiments were performed in the CNRS/Max-Planck-Institut High Magnetic Field Laboratory, Grenoble, under the project SE3394. Gy. K., B. P. and I.G. S. were also partially supported from grants No. 12037 and 14094 from the Hungarian National Research Fund (OTKA).

\section{References}

[1] A. Aoki, J. Phys. C 12, 633 (1979).

[2] H. Buhmann, W. Joss, K.v. Klitzing, I.V. Kukushkin, A.S. Plaut, G. Martinez, K. Ploog, V.B. Timofeev, Phys. Rev. Lett. 66, 926 (1991).

[3] V.J. Goldman, M. Santos, M. Shayegan, J.E. Cunningham, Phys. Rev. Lett. 65, $2189(1990)$.

[4] E.Y. Andrei, G. Deville, D.C. Glattli, F.I.B. Williams, E. Paris, B. Etienne, Phys. Rev. Lett. 66, 2765 (1988).

[5] B. Pödör, S.V. Novikov, I.G. Sa velev, G. Gombos, Acta Phys. Hung. 74, 143 (1994).

[6] Gy. Kovacs, V. Novikov, G. Gombos, B. Pödör, Gy. Remenyi, Acta Phys. Pol. A 87, 473 (1995).

[7] A.M. Kreshchuk, S.V. Novikov, I.G. Savel'ev, Fiz. Tekh. Poluprov. 26, 1375 (1992) [Sov. Phys. Semicond. 26, 771 (1992)].

[8] S.D. Bystrov, A.M. Kreshchuk, S.V. Novikov, T.A. Polyanskaya, I.G. Savel'ev, Fiz. Tekh. Poluprov. 27, 645 (1993) [Semiconductors 27, 358 (1993)]. 